\title{
Validación del Cuestionario de evaluación de la calidad de cursos virtuales adaptado a MOOC ${ }^{1}$ Validation of the Questionnaire of Quality Assessment of Online Courses adapted to MOOC
}

Santiago Mengual-Andrés

Carmen Lloret Catalá

Universidad de Valencia (España)

Rosabel Roig Vila

Universidad de Alicante (España)

\section{Resumen}

Los Massive Online Open Courses (MOOC) son una modalidad de formación virtual que está ya presente en el panorama educativo actual y que responde a una concepción determinada de e-learning. Las universidades están integrando como parte de su oferta formativa este tipo de formación o, al menos, se está debatiendo si se integra o no. Ante esto, se hace necesario calibrar cuáles deben ser sus características con el fin de configurar un tipo de formación virtual de calidad. Se considera que son necesarios instrumentos que avalen la calidad de los MOOC desde diferentes perspectivas, en este caso la pedagógica, ya que de ello dependerá el éxito y la consolidación de este tipo de e-learning. El objetivo del presente estudio ha sido, pues, validar un cuestionario para la evaluación pedagógica de los MOOC adaptando el cuestionario de evaluación de cursos virtuales (Arias, 2007). Se ha analizado la validez y fiabilidad de dicho cuestionario a través de un análisis factorial de componentes principales con rotación Varimax. Se concluye que bastaría con introducir adaptaciones en la relación de dimensiones propuestas en este cuestionario que tuviera en cuenta las especificidades de los MOOC, ya que estos no pueden analizarse única y exclusivamente desde la óptica general del e-learning anterior a los MOOC. Así, las dimensiones en las cuales se basa dicho cuestionario son: 1) la calidad de la comunicación y los elementos multimedia de los cursos masivos en 
línea; 2) la coherencia curricular de los cursos y el grado de adaptación al usuario y 3) la calidad de su planificación didáctica.

Palabras clave: MOOC; práctica pedagógica; educación en línea; investigación en educación a distancia; evaluación.

\begin{abstract}
Massive Online Open Courses (MOOC) constitute a virtual training modality which is already present at today's educational scenario, and which reflects a specific conception of e-learning. Universities are integrating this type of training as part of their formative offer or, at least, are debating whether to integrate it or not. In the light of the above, it becomes necessary to gauge what the characteristics of these courses should be so that they can configure a high-quality virtual training format. These are considered to be necessary instruments when it comes to guaranteeing the quality of MOOC from different perspectives, the pedagogical one in this case -since the success and consolidation of this e-learning format will depend thereon. The aim sought with the present study was therefore to validate a questionnaire for the pedagogical evaluation of MOOC through an adaptation of the cuestionario de evaluación de cursos virtuales [questionnaire for the evaluation of virtual courses] (Arias, 2007). The validity and reliability of the said test was checked using a principal components factor analysis with Varimax rotation. The conclusion drawn is that it would suffice to introduce adaptations on the list of dimensions proposed in this questionnaire that take into account the specificities of MOOC, since the latter cannot be exclusively analyzed from the general perspective of e-learning -as it was prior to MOOC. Thus, the dimensions which serve as the basis for that questionnaire are: 1) quality of communication and multimedia elements in online massive courses; 2) curricular consistency in such courses as well as their degree of adaptation to the user; and 3) quality of their didactic planning.
\end{abstract}

Keywords: MOOC; pedagogical practice; online education; review of distance education research; evaluation.

Las siglas MOOC, cuyo significado es Massive Open Online Course, se han convertido en un término difícil de separar, a su vez, de uno más general como es el e-learning. Cuando en 2012 se proclamaba dicho periodo como el año de los MOOC (Pappano, 2012) se estaba postulando una situación vinculada, no únicamente a ese corto periodo de tiempo en el cual hubo una verdadera explosión de iniciativas en 
torno a la organización de este tipo de cursos (Prendes y Sánchez, 2014), sino a la irrupción de una nueva modalidad de e-learning con una trayectoria que, desde entonces, se está trazando y se trazará en los próximos -al menos- años (Torres y Gago, 2014).

Es importante, pues, delimitar las características que debe tener este tipo de formación ya que, además, la Enseñanza Superior se ha implicado - casi de forma generalizada- en la oferta de MOOC como modalidad de enseñanza virtual de masas a bajo coste (Capdevila y Aranzadi, 2014) y en abierto (Duart y Mengual-Andrés, 2014; Yuan y Powell, 2013) y es que, tal y como apuntan Martín, González y García (2013) este tipo de formación resulta "extraordinariamente sugerente" y, en el caso, por ejemplo, de plataformas de MOOC no anglosajonas, como Miríada X, como "una gran oportunidad para satisfacer la demanda de los cientos de millones de hispano y luso parlantes". Algunos autores, pues, consideran como disruptivo el fenómeno MOOC (Christensen y Overdorf, 2000). En este sentido, los MOOC son considerados como la panacea para la democratización de la formación a través de la Red, además a muy bajo coste y de calidad. De estas tres cuestiones queremos abordar esta última y es que diversos autores apuntan las dudas acerca de ello, ya que "no parece tan evidente que cursos de estas características ofrezcan una formación de calidad" (Martín et al., 2013).

La calidad ha sido uno de los aspectos que con mayor énfasis se ha abordado en la trayectoria del e-learning: desde los formatos previos a ello, como era la educación a distancia cuando no existía Internet, hasta los formatos actuales (Bates y Sangrà, 2012; Sánchez, Tomás, Serrano y Prendes, 2013), donde los recursos y posibilidades de conexión, información y comunicación de la Red, junto a los dispositivos móviles (Anderson, 2013) están abriendo nuevos escenarios de aprendizaje. En esta trayectoria se han ido sucediendo cambios, nuevos planteamientos e hitos que han marcado mejoras y adaptaciones al contexto (Sánchez, Tomás, Serrano y Prendes, 2013), llegando a posibilidades de modelos de formación virtual donde la combinación de los recursos para la información, la comunicación y el aprendizaje están dando lugar actualmente a propuestas muy interesantes (Castaño, Maiz y Garay, 2015). 
El e-learning, pues, ha evolucionado atendiendo a nuevos parámetros pedagógicos que han superado, en cada momento, carencias, limitaciones y posibilidades de la situación anterior (Alvarado, 2014; Ardila-Rodríguez, 2011; Najmul, 2013; Torres y Ortega, 2003; Vidal y Camarena, 2014), tal y como lo hacen otros conceptos, modelos y temáticas en el ámbito pedagógico. De este modo, los MOOC son actualmente el último estadio en la evolución del e-learning y se plantean, así, propuestas impensables hace años, como es el caso de un mobiMOOC (DeWaard, Abajian, Gallagher, Hogue, Keskin, Koutropoulos y Rodríguez, 2011). De este forma, se han definido incluso los MOOC como un fenómeno disruptivo per se (Barber, Donnelly, Rizvi y Summers, 2013; Christensen y Overdorf, 2000; Skiba, 2012), lo cual, consideramos es excesivo sin hacer previamente una evaluación exhaustiva de dicho fenómeno. En este sentido, no todos los autores defienden esta exacerbada consideración (Conole, 2013), lo cual compartimos. Es cierto que, de forma global, los MOOC están consiguiendo millones de alumnos matriculados, con lo que eso conlleva de democratización del conocimiento. Ahora bien, consideramos que la irrupción de los MOOC responde a una "efervescencia" (Roig, Mengual-Andrés y Suárez, 2014) más en el ámbito pedagógico.

Lo realmente importante es evaluar esta irrupción y analizar la calidad de este tipo de formación por dos motivos: en primer lugar, para verificar que realmente es una evolución en la trayectoria del modelo del e-learning y que no supone una involución de prácticas pedagógicas que ya estaban superadas y, por otro lado, para verificar que este nuevo modelo reúne una serie de características que avalan la calidad del mismo y, en caso de no ser así, cómo poder abordar su optimización (Guàrdia, Maina y Sangrà, 2013).

Consideramos, pues, que la calidad pedagógica de los MOOC debe ser una cuestión fundamental en este tipo de formación y que, desgraciadamente, al encontrarnos en el momento de su "efervescencia", no hay suficientes estudios que hagan posible un acuerdo generalizado acerca de qué criterios de calidad deben primar en los MOOC. Así lo apuntan Bates (2014), Martín et al. (2013) y Read 
y Rodrigo (2014). Estos últimos, de hecho, evaluaron los MOOC de la primera edición de UNED COMA, que se desarrollaron entre octubre de 2012 y mayo de 2013, a partir de un prototipo de modelo y que respondía más bien a una solución práctica para realizar en dicho momento la evaluación pertinente de los cursos, que a un modelo fundamentado y experimentado, como así lo afirman los autores.

En un trabajo anterior (Roig et al., 2014) exponíamos los resultados de una investigación acerca de la calidad pedagógica de 52 MOOC. Además, apuntábamos algunos aspectos investigados acerca de los MOOC no directamente relacionados con aspectos pedagógicos: certificaciones (Daniel, 2012) y análisis de mercado (McAuley, Stewart, Siemens y Cormier, 2010; Vega, 2013); otros relacionados con el conocimiento abierto (Anderson, 2013); otros relacionados de una forma u otra con aspectos pedagógicos: educación formal y no formal (Kop, Fournier y Mak, 2011), cambios en la Enseñanza Superior (Dennis, 2012; Lombillo, López y Zumeta, 2012) y tasas de participación y abandono (Mackness, Mak y Williams, 2010), y otros directamente referidos a cuestiones pedagógicas (Giorgetti, Romero y Vera, 2013; Glance, Forsey y Riley, 2013; Martín et al., 2013; Vázquez, López y Sarasola, 2013; Zapata, 2013).

Es importante reseñar nuevas referencias interesantes (Bates, 2014; Castaño et al., 2015; Gasevic, Kovanovic, Joksimovic y Siemens, 2014; Yousef, Chatti, Schroeder, Wosnitza y Jakobs, 2014). De todas ellas, merece especial atención la iniciativa Mooc Quality Project de la European Foundation for Quality e-Learning (EFQUEL) (http:// mooc.efquel.org/), que aborda la cuestión de la calidad de los MOOC, pero sin tratar de encontrar una respuesta única sino estimular un discurso sobre dicho tema a partir de una serie de publicaciones en su web por parte de expertos y empresarios de todo el mundo en el campo donde abordan esta cuestión desde su punto de vista. Otra iniciativa importante es la OpenupEd (Rosewell y Jansen, 2014), con la que se pretende definir una marca de calidad -denominada OpenupEd Quality Label- para ofrecer un instrumento de evaluación, especialmente referido a lo abierto y el e-learning, pero aplicado específicamente alos MOOC. Con ello, las universidades que pertenecen 
a esta Asociación Internacional pretenden ofrecer un modelo para evaluar la calidad de los MOOC, tanto a nivel institucional como a nivel particular del curso ofertado. Se definió en enero de 2014, a partir de la E-xcellence label (Williams, Kear y Rosewell, 2012), la cual se aplicaba para evaluaciones institucionales de e-learning y blended learning. OpenupEd se trata del primer proyecto de este tipo que se desarrolla con apoyo de la Comisión Europea (Read y Rodrigo, 2014) y de la que forman parte universidades de doce países, entre las que se encuentra la UNED, The Open University y Open University of the Netherlands. En este sentido, en el contexto español se ha aplicado la OpenupEd Quality Label para evaluar MOOC de UNED COMA (Rodrigo, Read, Santamaría y Sánchez-Elvira, 2014), pero hay algunas cuestiones, como apuntan los autores, tales como el modelo concreto de diseño instruccional, el apoyo a los estudiantes con nuevos perfiles, el reconocimiento de la carga de trabajo del profesor, etc. que merecen seguir investigando sobre propuestas de modelos para evaluar la calidad de los MOOC.

En este sentido, en el presente trabajo queremos presentar un instrumento de evaluación con suficientes garantías de validación que pueda ser utilizado y complemente el panorama actual sobre el cual construir un modelo ecléctico para la evaluación de la calidad pedagógica de los MOOC. En definitiva, tal y como indicábamos en un trabajo anterior, "los MOOC están vinculados, de una forma u otra, a la formación a través de la Red en el contexto actual” (Roig et al., 2014), y lo fundamental ahora es determinar qué calidad debe tener dicha formación. Así pues, el objetivo del presente estudio ha sido validar un cuestionario para la evaluación pedagógica de los MOOC adaptando el cuestionario de evaluación de cursos virtuales (Arias, 2007), como propuesta para tal menester. 


\section{METODOLOGÍA}

\section{Participantes}

En este estudio se han analizado 52 cursos MOOC de temática perteneciente al ámbito de las Ciencias Sociales y ofertados por 10 plataformas distintas (Miríada X, Coursera, Udacity, UniMOOC, OpenLearning, OpenHPI, UNED COMA, Class2Go, Canvas Network, EdX). Para su selección se ha realizado un muestreo no probabilístico disponible (McMillan y Shumacher, 2005). El 50\% $(n=26)$ de los cursos analizados empleaban el inglés como lengua vehicular y otro 50\% $(n=26)$ el español, y todos ellos estaban activos en abril de 2013. Los datos recogidos se emplearían posteriormente en el proceso de validación del instrumento. La evaluación de dichos cursos ha sido realizada por 5 jueces expertos, con un perfil de profesor titular de universidad y especialista en Tecnología Educativa, y cuya selección fue intencional (Bisquerra, 2004).

\section{Procedimiento}

Fruto de las conclusiones del estudio de Roig et al. (2014) se estableció la necesidad de construir un instrumento de evaluación de la calidad pedagógica de los MOOC basado en las particularidades de este tipo de cursos y con un número de dimensiones sensiblemente inferior a las propuestas en el instrumento original, a saber, el Cuestionario de evaluación de la calidad de cursos virtuales de Arias (2007).

Se diseñó una versión electrónica del cuestionario con el software Limesurvey. Se procedió a dar de alta a los evaluadores en la plataforma y se remitió el posterior contacto por correo electrónico con la indicación de las direcciones web de los cursos objeto de evaluación, el enlace al cuestionario y las indicaciones necesarias para realizar las evaluaciones. Fue necesario, para ello, darse de alta en los MOOC analizados, lo cual se hizo con una cuenta de usuario común. Una vez finalizado el proceso de recogida de información, fueron exportados los datos brutos a un fichero csv para su posterior importación y tratamiento estadístico. 


\section{Instrumento}

Para llevar a cabo este estudio era necesario un instrumento con el cual poder realizar una evaluación de los MOOC desde una perspectiva pedagógica. Por ello, se consideró adecuado utilizar el Cuestionario de evaluación de la calidad de cursos virtuales (Arias, 2007). Dicho instrumento está formado por 36 ítems agrupados en 10 dimensiones o factores, los cuales se expresan en una escala Likert de 5 puntos (1 = nunca; 2 = casi nunca, $3=$ a veces, $4=$ casi siempre, $5=$ siempre), excepto los pertenecientes a la primera dimensión y los ítems 14,16 y 13 que presentaban una escala Likert de cuantificación. Así, dichas dimensiones son:

1. Guía didáctica: 4 ítems dicotómicos (sí/no) matizan las pretensiones del curso, objetivos y actividades de refuerzo.

2. Metodología: 4 ítems que indagan la adecuación de los contenidos a los objetivos, la coherencia metodológica y la coherencia de las actividades de refuerzo.

3. Organización de los contenidos: 3 ítems que valoran la estructura de los contenidos.

4. Calidad de los contenidos: 7 ítems que evalúan el grado de adecuación de los conocimientos que se explican dentro del curso.

5. Recursos didácticos: 4 ítems alusivos a la versatilidad del curso para enseñar el contenido de formas distintas.

6. Capacidad de motivación: 1 ítem que evalúa el grado de motivación del curso.

7. Elementos multimedia: 7 ítems que valoran la calidad multimedia y gráfica del curso y sus recursos.

8. Estilo del lenguaje: 2 ítems que analizan la sintaxis y la semántica de los contenidos.

9. Discriminación y valores: 2 ítems que evalúan la adecuación de los contenidos a unas normas éticas y valores aceptados universalmente. 
10. Singularidad del usuario: 2 ítems que evalúan el grado de adaptación del MOOC a las particularidades del estudiante.

\section{Análisis de datos}

Se empleó el paquete estadístico SPSS 22 con el objeto de determinar la estructura factorial a través del análisis factorial exploratorio. Se comprobó la adecuación muestra (KMO) y se determinó el tamaño idóneo de los componentes principales.

\section{RESULTADOS}

\section{Validez de constructo}

Con objeto de esclarecer la pertinencia y posibilidad de un análisis factorial en base a los datos obtenidos a través del cuestionario original se verificó la adecuación muestral KMO (Kaiser-Meyer-Olkin). Los resultados evidenciaron una bondad de ajuste aceptable con un coeficiente superior a .5. Los resultados de la prueba de esfericidad de Barlett $(\mathrm{p}=.000)$ evidenciaron que la matriz de correlación es distinta a la matriz de identidad. Los resultados anteriores demuestran la idoneidad de la aplicación del análisis factorial.

La bondad del ajuste es aceptable, justificando el análisis factorial, dado que la medida de adecuación muestral de Kaiser-Meyer-Olkin (KMO) toma un valor superior a 0.5 , y la prueba de esfericidad de Bartlett evidencia por tanto que la matriz de correlación es distinta a la matriz de identidad (tabla 1).

Tabla 1. KMO y Test de Bartlett

\begin{tabular}{|c|c|c|}
\hline \multicolumn{2}{|c|}{$\begin{array}{c}\text { Kaiser-Meyer-Olkin Measure of Sampling Ade- } \\
\text { quacy. }\end{array}$} & .555 \\
\hline \multirow{2}{*}{$\begin{array}{c}\text { Bartlett's Test of } \\
\text { Sphericity }\end{array}$} & Approx. Chi-Square & 1356.334 \\
\cline { 2 - 3 } & $\mathrm{df}$ & 630 \\
\cline { 2 - 3 } & Sig. & .000 \\
\hline
\end{tabular}


Se prosiguió a la extracción de los factores a través de un análisis factorial de componentes principales y rotación Varimax. Los resultados de la regla Kaiser evidenciaron 10 componentes principales que explicaron el 80.55\% de la varianza total (Ver tabla 2).

Tabla 2. Varianza total explicada

\begin{tabular}{|l|c|c|c|c|c|c|}
\hline \multirow{2}{*}{ Componente } & \multicolumn{3}{|c|}{ Autovalores iniciales } & \multicolumn{2}{c|}{$\begin{array}{c}\text { Sumas de las saturaciones al } \\
\text { cuadrado de la extracción }\end{array}$} \\
\cline { 2 - 7 } & Total & $\begin{array}{c}\text { \% de la } \\
\text { varianza }\end{array}$ & $\begin{array}{c}\text { \% } \\
\text { Acumulado }\end{array}$ & Total & $\begin{array}{c}\text { \% de la } \\
\text { varianza }\end{array}$ & $\begin{array}{c}\text { \% } \\
\text { Acumulado }\end{array}$ \\
\hline 1 & 11.591 & 32.197 & 32.197 & 11.591 & 32.197 & 32.197 \\
\hline 2 & 3.816 & 10.6 & 42.797 & 3.816 & 10.6 & 42.797 \\
\hline 3 & 2.985 & 8.291 & 51.088 & 2.985 & 8.291 & 51.088 \\
\hline 4 & 2.04 & 5.667 & 56.755 & 2.04 & 5.667 & 56.755 \\
\hline 5 & 1.882 & 5.227 & 61.983 & 1.882 & 5.227 & 61.983 \\
\hline 6 & 1.615 & 4.487 & 66.47 & 1.615 & 4.487 & 66.47 \\
\hline 7 & 1.443 & 4.009 & 70.479 & 1.443 & 4.009 & 70.479 \\
\hline 8 & 1.274 & 3.539 & 74.018 & 1.274 & 3.539 & 74.018 \\
\hline 9 & 1.213 & 3.369 & 77.386 & 1.213 & 3.369 & 77.386 \\
\hline 10 & 1.137 & 3.159 & 80.546 & 1.137 & 3.159 & 80.546 \\
\hline
\end{tabular}

En una lectura aproximativa de la matriz de componentes rotados se observa que la cantidad de componentes se ajusta a los propuestos inicialmente por la estructura del cuestionario original. Sin embargo, la observación detallada evidenció que numerosos reactivos no se situaban en las dimensiones propuestas. La lectura del gráfico de sedimentación (figura 1) reforzó la idea de proponer otra agrupación de ítems, mostrando un fuerte cambio de pendiente a partir del cuarto componente. 
Figura 1. Gráfico de sedimentos

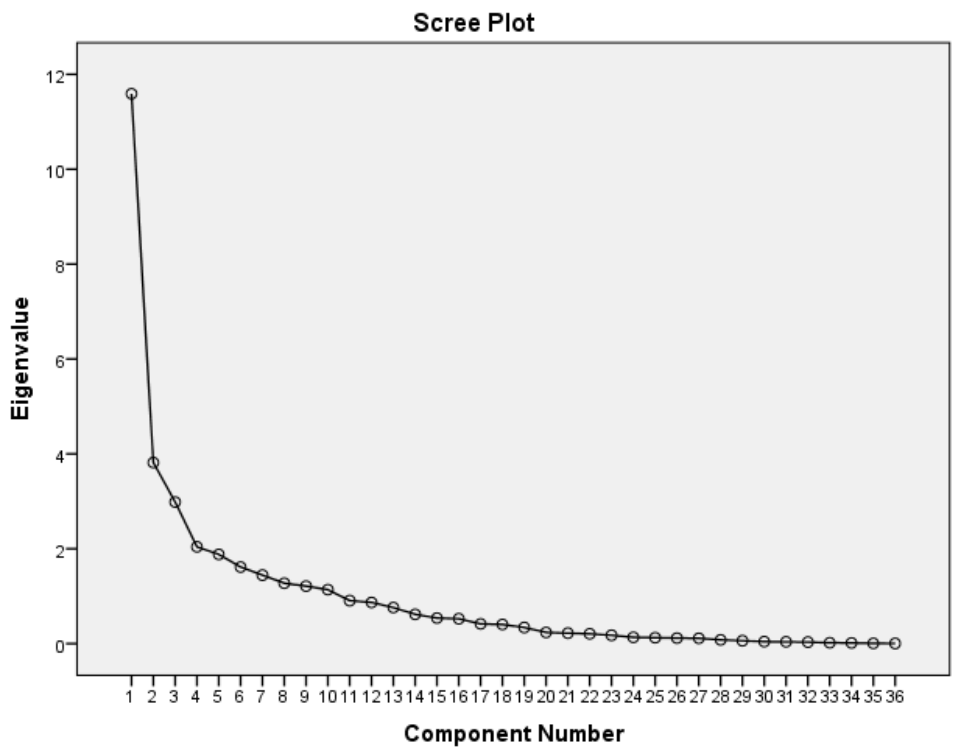

Esta lectura condujo al análisis de los ítems en función de su saturación con otras dimensiones. Se procedió a realizar los ajustes y extraer únicamente los tres componentes principales a través de un análisis de segundo orden. Se verificó la adecuación muestral (KMO) y la prueba Barlett. Los resultados evidenciaron valores aceptables con puntuaciones de .60 y .ooo respectivamente.

La extracción de los factores a través del método de componentes principales y rotación Varimax obtuvo un resultado de tres factores: a) factor uno: calidad en la comunicación y elementos multimedia (ítems: 28, 30, 29, 27, 26, 25, 24, 22, 17, 21, 23 y 31); b) factor dos: coherencia curricular y adaptación al usuario (ítems: 5, 32, 35, 6, 4, 10, 20, 34 y 15); c) factor tres: planificación didáctica (ítems: 8, 7, 14, 18, 19, $33,12,16,1,3,2,11$ y 13). Se obtuvieron autovalores mayores que 1.00 (11.59, 3.82 y 2.98, respectivamente), explicando una varianza total del 85,09\% (32,20\%, 30.6\%, 22.29\%) (tabla 3). 
Tabla 3. Método de extracción: análisis de componentes principales

\begin{tabular}{|c|c|c|c|c|}
\hline & \multirow[t]{2}{*}{ Ítem } & \multicolumn{3}{|c|}{ Componente } \\
\hline & & 1 & 2 & 3 \\
\hline 28. & $\begin{array}{l}\text { ¿Es buena la calidad de las presentaciones } \\
\text { audiovisuales? }\end{array}$ & ,878 & & \\
\hline 30. & ¿Es buena la calidad de mensajes de audio? & ,865 & & \\
\hline 29. & $\begin{array}{l}\text { ¿Se adecuan las presentaciones audiovisuales } \\
\text { al texto? }\end{array}$ & ,862 & & \\
\hline 27. & ¿Se adecuan las animaciones al texto? & ,848 & & \\
\hline 26. & ¿Es buena la calidad de las animaciones? & ,839 & & \\
\hline 25. & ¿Se adecuan las imágenes y gráficos al texto? & ,737 & & \\
\hline 24. & ¿Es buena la calidad de las imágenes? & ,686 & & \\
\hline 22. & $\begin{array}{l}\text { ¿Se puede recurrir a un Sistema de ayudas y } \\
\text { refuerzos constantes sobre los contenidos? }\end{array}$ & ,690 &, 329 & \\
\hline 17. & $\begin{array}{l}\text { ¿La comunicación del programa con el usuario } \\
\text { es interactiva? }\end{array}$ &, 783 & ,495 & 314 \\
\hline 21. & $\begin{array}{l}\text { ¿Se emplean diversos códigos comunicativos } \\
\text { (verbal, icónico, etc.?) }\end{array}$ &, 777 & ,446 & \\
\hline 23. & $\begin{array}{l}\text { ¿Las actividades propuestas en el sistema } \\
\text { resultan atractivas? }\end{array}$ & ,693 & & 313 \\
\hline 31. & $\begin{array}{l}\text { ¿Es correcta la ortografía, corrección gramatical } \\
\text { y sintáctica del texto? }\end{array}$ & ,690 & & ,467 \\
\hline 9. & $\begin{array}{l}\text { ¿Está actualizada la información que se } \\
\text { muestra? }\end{array}$ &, 519 & & \\
\hline $5 \cdot$ & $\begin{array}{l}\text { ¿Los contenidos responden a los objetivos } \\
\text { planteados? }\end{array}$ & & ,804 & \\
\hline 32. & $\begin{array}{l}\text { ¿El lenguaje usado está adaptado al nivel del } \\
\text { usuario potencial? }\end{array}$ & & ,797 & \\
\hline 35. & $\begin{array}{l}\text { ¿El sistema contempla las características y } \\
\text { circunstancias personales y/o particulares de } \\
\text { los usuarios (incluida alguna discapacidad)? }\end{array}$ & &, 763 & \\
\hline 6. & $\begin{array}{l}\text { ¿La evaluación es coherente con la metodología } \\
\text { planteada? }\end{array}$ & ,389 &, 700 & \\
\hline 4. & $\begin{array}{l}\text { ¿Las actividades son coherentes con la } \\
\text { metodología planteada? }\end{array}$ & & ,894 & \\
\hline 10 & $\begin{array}{l}\text { ¿La relación entre lo fundamental y lo accesorio } \\
\text { en la información está claramente definida? }\end{array}$ & &, 713 & ,431 \\
\hline
\end{tabular}




\begin{tabular}{|c|c|c|c|c|}
\hline & Ítem & Comp & ente & \\
\hline & & 1 & 2 & 3 \\
\hline 20. & $\begin{array}{l}\text { ¿Los conceptos nuevos se introducen mediante } \\
\text { esquemas, resúmenes, síntesis? }\end{array}$ &, 330 &, 704 &, 301 \\
\hline 34. & $\begin{array}{l}\text { ¿Los textos y los contenidos cumplen el } \\
\text { mandato constitucional de no hacer distinción } \\
\text { o discriminación por razón de nacimiento, } \\
\text { raza, sexo, religión, opinión o cualquier otra } \\
\text { condición o circunstancia personal o social? }\end{array}$ & &, 542 &, 314 \\
\hline 15 . & $\begin{array}{l}\text { ¿Los contenidos se presentan de forma } \\
\text { hipertextual? }\end{array}$ &, 324 & ,667 & ,364 \\
\hline 8. & $\begin{array}{l}\text { ¿Las actividades de refuerzo, cuando las hay, } \\
\text { permiten superar las posibles deficiencias que } \\
\text { se han detectado en la evaluación? }\end{array}$ & & & ,773 \\
\hline 7. & ¿Existen actividades de refuerzo? & & &, 760 \\
\hline 14. & $\begin{array}{l}\text { El número de secuencias o itinerarios de repaso } \\
\text { es: [1- no hay/2- bajo/3-suficiente/4-alto/5- } \\
\text { muy alto] }\end{array}$ & & ,470 &, 714 \\
\hline 18. & $\begin{array}{l}\text { ¿Se plantean actividades abiertas que fomenten } \\
\text { la creatividad? }\end{array}$ & ,484 & &, 681 \\
\hline 19. & $\begin{array}{l}\text { ¿El sistema dispone de distintos itinerarios de } \\
\text { aprendizaje? }\end{array}$ & ,461 & & ,773 \\
\hline 33. & $\begin{array}{l}\text { En general, ¿̇los contenidos de los mensajes de } \\
\text { interacción con el usuario son positivos? }\end{array}$ & &, 510 &, 634 \\
\hline 12. & $\begin{array}{l}\text { ¿Existen distintos niveles de contenidos en } \\
\text { función de los usuarios? }\end{array}$ & & & ,477 \\
\hline 16. & $\begin{array}{l}\text { Para conseguir los objetivos planteados el } \\
\text { número de actividades es: [1- no hay/2-bajo/3- } \\
\text { suficiente/4-alto/5-muy alto] }\end{array}$ & &, 305 &, 655 \\
\hline 1. & ¿Existe la guía didáctica del sistema? & & & ,447 \\
\hline 3. & ¿Los objetivos se plantean explícitamente? & & ,365 &, 788 \\
\hline 2. & $\begin{array}{l}\text { ¿En la guía didáctica (si existe) se expresa } \\
\text { claramente cómo integrar el sistema en el } \\
\text { proceso de enseñanza-aprendizaje? }\end{array}$ & & & ,426 \\
\hline 11. & ¿Se ofrece una buena selección bibliográfica? & & & ,424 \\
\hline
\end{tabular}




\begin{tabular}{|l|l|l|l|l|}
\hline & Ítem & \multicolumn{3}{|l|}{ Componente } \\
\cline { 3 - 5 } & & $\mathbf{1}$ & $\mathbf{2}$ & $\mathbf{3}$ \\
\hline 13. & $\begin{array}{l}\text { El número de actividades propuestas para } \\
\text { realizar en grupo es: [1- no hay/2- bajo/3- } \\
\text { suficiente/4-alto/5-muy alto] }\end{array}$ & &, 377 \\
\hline 36. & $\begin{array}{l}\text { El alumno puede organizar su tiempo de } \\
\text { estudio en horarios flexibles }\end{array}$ & & 0,29 \\
\hline & Varianza explicada por cada factor & $32,20 \%$, & $30.6 \%$, & $22.29 \%$ \\
\hline & \multicolumn{3}{|c|}{ Varianza total explicada } & \multicolumn{3}{|c|}{$8 \%$} \\
\hline
\end{tabular}

Los resultados de la nueva extracción de componentes y la lectura de los reactivos evidenciaron que la nueva agrupación de ítems resultaba más acorde y simplificada, pasando de los 36 ítems originales a 34. El leve aumento de la varianza total explicada respecto a la agrupación en los factores originales también evidenciaba dicha pertinencia.

Análisis de consistencia interna

El instrumento original (Arias, 2007) poseía una validez de contenido adecuada y un índice de fiabilidad elevado $(\alpha=.93)$. Los análisis de fiabilidad Alpha de Cronbach realizados en el presente estudio arrojaron para los 34 ítems resultantes una puntuación total de .95. En un análisis más específico la dimensión calidad en la comunicación y elementos multimedia obtuvo un índice .93, la dimensión coherencia curricular y adaptación al usuario un .90 y la dimensión planificación didáctica 90.

Se realizaron los pertinentes test de correlación ítem-total. Así, los resultados no evidenciaron la mejora de los índices de fiabilidad (totales y en cada dimensión) con la modificación o eliminación de ninguno de los ítems propuestos.

\section{Composición definitiva}

La composición definitiva del instrumento Cuestionario de evaluación de la calidad de cursos virtuales adaptado a MOOC quedó del siguiente modo: 
Dimensión 1: Calidad en la comunicación y elementos multimedia

1. ¿La comunicación del programa con el usuario es interactiva?

2. ¿Se emplean diversos códigos comunicativos (verbal, icónico, etc.)?

3. ¿̇e puede recurrir a un sistema de ayudas y refuerzos constantes sobre los contenidos?

4. ¿Las actividades propuestas en el sistema resultan atractivas?

5. ¿Es buena la calidad de las imágenes?

6. ¿Se adecuan las imágenes y gráficos al texto?

7. ¿Es buena la calidad de las animaciones?

8. ¿Se adecuan las animaciones al texto?

9. ¿Es buena la calidad de las presentaciones audiovisuales?

10. ¿Se adecuan las presentaciones audiovisuales al texto?

11. ¿Es buena la calidad de mensajes de audio?

12. ¿Es correcta la ortografía, corrección gramatical y sintáctica del texto?

Dimensión 2: Coherencia curricular y adaptación al usuario

1. ¿Las actividades son coherentes con la metodología planteada?

2. ¿Los contenidos responden a los objetivos planteados?

3. ¿̇La evaluación es coherente con la metodología planteada?

4. ¿̇a relación entre lo fundamental y lo accesorio en la información está claramente definida?

5. ¿Los contenidos se presentan de forma hipertextual?

6. ¿Los conceptos nuevos se introducen mediante esquemas, resúmenes, síntesis?

7. ¿El lenguaje usado está adaptado al nivel del usuario potencial?

8. ¿El sistema contempla las características y circunstancias personales y/o particulares de los usuarios (incluida alguna discapacidad)? 
9. ¿Los textos y los contenidos cumplen el mandato constitucional de no hacer distinción o discriminación por razón de nacimiento, raza, sexo, religión, opinión o cualquier otra condición o circunstancia personal o social?

\section{Dimensión 3: Planificación didáctica}

1. ¿Existe la guía didáctica del sistema?

2. ¿En la guía didáctica (si existe) se expresa claramente cómo integrar el sistema en el proceso de enseñanza-aprendizaje?

3. ¿Los objetivos se plantean explícitamente?

4. ¿Las actividades de refuerzo, cuando las hay, permiten superar las posibles deficiencias que se han detectado en la evaluación?

5. ¿Existen actividades de refuerzo?

6. El número de secuencias o itinerarios de repaso es: [1-no hay/2bajo/3-suficiente/4-alto/5-muy alto]

7. ¿Se plantean actividades abiertas que fomenten la creatividad?

8. ¿El sistema dispone de distintos itinerarios de aprendizaje?

9. ¿Existen distintos niveles de contenidos en función de los usuarios?

10. Para conseguir los objetivos planteados el número de actividades es: [1-no hay/2- bajo/3-suficiente/4-alto/5-muy alto]

11. En general, ¿̇los contenidos de los mensajes de interacción con el usuario son positivos?

\section{DISCUSIÓN Y CONCLUSIONES}

Las investigaciones y discusiones sobre los MOOC se han centrado en el potencial social, institucional, tecnológico o cuestiones de marketing y menos en la calidad de los mismos (Yousef et al., 2014). Cuando diversos estudios han apuntado una tasa de deserción en los MOOC en torno al 95\% de los participantes y otros problemas pedagógicos relativos a la evaluación y retroalimentación (Prendes y Sánchez, 2014), se hace necesario abordar la calidad pedagógica 
en este tipo de e-learning. En el estudio realizado por Yousef et al. (2014) donde se analizan 84 publicaciones sobre MOOC se concluye que los principales temas abordados en las mismas son: concepto (20 publicaciones), diseño (16), estudios de caso (15), teorías de aprendizaje (15) y modelos de negocio (8). Aunque sobre diseño aparecen 16 publicaciones, ahondando en el análisis de las mismas, no se hace referencia a la calidad pedagógica en ninguna de ellas, sino más bien en la descripción del diseño utilizado en la oferta correspondiente de diversos MOOC.

Se hace necesario, pues, abordar estudios sobre la calidad pedagógica de los MOOC. En este sentido, el análisis de los mismos puede abordarse desde la óptica genérica, a partir de instrumentos de evaluación pensados para los cursos virtuales, o desde una óptica específica. Desde nuestra lectura, los MOOC, aun siendo una tendencia dentro del e-learning, nacen en otro contexto y poseen características que los hacen distintos de los cursos virtuales tradicionales. Es por ello que, entre ambos tipos de modelos, existen elementos comunes y elementos distintos, y como tales deben ser evaluados. En este sentido, coincidimos con Yousef et al. (2014) cuando indica que, aunque se hayan realizado varios estudios identificando un gran conjunto de criterios para el éxito del diseño de cursos de e-learning en general, no todos ellos se pueden utilizar en los MOOC, debido a algunas características únicas de los mismos.

Cabe pensar, pues, que la evaluación pedagógica de los MOOC a partir de unas dimensiones pensadas para los entornos virtuales de aprendizaje más tradicionales son poco acertados. El presente trabajo ha pretendido, en base a un instrumento ya validado (Arias, 2007) establecer un modelo de evaluación de la calidad pedagógica de los MOOC, el cual queda definido por tres dimensiones: 1) la calidad de la comunicación y los elementos multimedia de los cursos masivos en línea; 2) la coherencia curricular de los cursos y el grado de adaptación al usuario y 3) la calidad de su planificación didáctica.

La propuesta de otras dimensiones entra en confrontación con la propia filosofía del MOOC: la "democratización” del acceso al conocimiento a través de la sencillez de los cursos, sus materiales y 
sus propuestas de comunicación. Así, de igual forma concurrimos con los resultados obtenidos por Castaño et al. (2015) según los cuales un diseño basado en la calidad de la comunicación, concretamente en las redes sociales, puede ayudar a reducir la tasa de abandono.

Los resultados del presente trabajo evidencian la fiabilidad de lo que podríamos denominar Cuestionario de evaluación de la calidad de cursos virtuales adaptado a MOOC, es decir, la adaptación del Cuestionario de evaluación de la calidad de cursos virtuales (Arias, 2007) al análisis de los Massive Online Open Courses. Los análisis de fiabilidad han presentado valores levemente superiores al cuestionario original y la redistribución de las dimensiones de 10 a 3 con la supresión de 3 ítems ha aumentado levemente la varianza total explicada. En este sentido, nuestros resultados coinciden con algunos de los principios que apunta Bates (2014) sobre cuáles deben regir el diseño de los MOOC, entre los que destacamos: comunicación, diversidad -por cuanto adaptación- y materiales de apoyo.

Con todo, queda puesto en evidencia que los MOOC como nueva expresión del e-learning no pueden analizarse únicamente y exclusivamente desde la óptica tradicional de los cursos virtuales. Existen, por supuesto, puntos de confluencia, como así lo demuestra el estudio realizado por Torres y Ortega (2003) -cuando "no existía” todavía la modalidad de formación MOOC como se entiende actualmente-, según el cual una dimensión importante era la calidad comunicacional. De igual forma, en el análisis que realizan Vidal y Camarena (2014) concurrimos en la importancia de la coherencia curricular -o contenido, como lo denominan estas autoras-.

Coincidimos respecto a esta visión específica de los MOOC frente a los modelos anteriores con la propuesta OpenupEd Quality Label (Rosewell y Jansen, 2014). Esta deriva de la E-xcellence label (Williams et al., 2012), cuyos indicadores también han sido adaptados para ser más apropiados al contexto del MOOC. Aun así, la propuesta OpenupEd Quality Label hace referencia a 21 indicadores institucionales y 11 específicos del curso. Nuestra propuesta se centra específicamente en el curso, sin atender a cuestiones institucionales, las cuales consideramos deben ser abordadas, pero 
en otro nivel. Aun así, podemos tomar como referencia la evaluación de Rodrigo et al. (2014) donde se aplica la OpenupEd Quality Label a diversos MOOC de COMA UNED. En este estudio el instrumento utilizado no pretende ser "una camisa de fuerza" sino una propuesta orientadora en la que unos MOOC se ajustarán a las características más que otros, pero todos se ajustarán en cierto grado, cuestión que creemos seráasía la hora de aplicar nuestra propuesta de instrumento de evaluación y que consideramos queda como limitación de nuestro estudio.

Actualmente no hay consenso sobre cómo evaluar la calidad de los MOOC (Rodrigo et al., 2014) y, además, es fundamental buscar indicadores de calidad con el fin de no sucumbir en modelos pedagógicos tradicionales aplicados ahora a cursos masivos abiertos online. Nos encontramos en "un momento en que las universidades se replantean su oferta formativa online" (Castaño et al., 2015) y es que, en el contexto tecnológico en el cual nos encontramos, es recomendable que las instituciones promuevan cursos virtuales en las respectivas ramas del conocimiento. Ahora bien, es necesario ofrecer formación de calidad, de lo contrario tenderá a desaparecer dicha oferta por falta de demanda, y necesario es también tener instrumentos de evaluación de dicha calidad, en especial desde una perspectiva pedagógica porque no olvidemos que, independientemente de las razones que mueven a las instituciones -tanto privadas como públicas- a ofrecer MOOC, estos tienen, como base, la educación.

\section{NOTAS}

1. El presente trabajo se enmarca en el seno del Grupo de Investigación "EDUTICADEI” (Ref.: VIGROB-039) y del Programa de Redes de investigación en docencia universitaria del ICE, todos ellos de la Universidad de Alicante; del proyecto "Instituto Superior de Investigación Cooperativa IVITRA" (Ref.: ISIC/2012/022; http://www.ivitra.ua.es) y del Proyecto DIGICOTRACAM ("Programa Prometeo de la Generalitat Valenciana para Grupos de Investigación en I+D de Excelencia”, Ref.: Prometeo-2009-042, cofinanciado por el FEDER de la UE y MICINN FFI2009-13065 y FFI2010-09064). 


\section{REFERENCIAS BIBLIOGRÁFICAS}

Alvarado García, M. A. (2014). Retroalimentación en educación en línea: una estrategia para la construcción del conocimiento. RIED. Revista Iberoamericana de Educación a Distancia, 17 (2), 5973. doi: $\underline{10.5944 / \text { ried.17.2.12678 }}$

Anderson, T. (2013). Promise and/or Peril: MOOC and Open and Distance Education. Commonwealth of Learning.

Ardila-Rodríguez, M. (2011). Indicadores de calidad de las plataformas educativas digitales. Educación y educadores, 14 (1), 189206. doi: 10.5294/edu.2011.14.1.10

Arias, J. (2007). Evaluación de la calidad de Cursos Virtuales: Indicadores de calidad $y$ construcción de un cuestionario de medida. Aplicación al ámbito de asignaturas de Ingeniería Telemática (tesis doctoral). Universidad de Extremadura, España.

Barber, M., Donnelly, K., Rizvi, S., y Summers, L. (2013). An avalanche is coming: Higher education and the revolution ahead. London, UK: Institute for Public Policy Research.

Bates, A. W., y Sangrà, A. (2012). La gestión de la tecnología en la educación superior. Estrategias para transformar la enseñanza y el aprendizaje. Barcelona: Octaedro.
Bates, T. (2014). Comparing $x M O O C$ and cMOOC: philosophy and practice.

Bisquerra, R. (2004). Metodología de la investigación educativa. Madrid: La Muralla.

Brinton, C., Chiang, M., Jain, S., Lam, H., Liu, Z., y Ming Fai Wong, F. (2013). Learning about social learning in MOOC: From statistical analysis to generative model. Ithaca: Cornell University Library.

Capdevila Pagès, R., y Aranzadi Elejabeitia, P. (2014). Los Cursos Online Masivos y Abiertos: ¿oportunidad o amenaza para las universidades iberoamericanas? RIED. Revista Iberoamericana de Educación a Distancia, 17 (1), 6982. doi: $10.5944 /$ ried.17.1.11574.

Castaño, C., Maiz, I., y Garay, U. (2015). Diseño, motivación y rendimiento en un curso MOOC cooperativo. Comunicar, 44. doi: 10.3916/C442015-02

Conole, G. (2013). Los MOOC como tecnologías disruptivas: estrategias para mejorar la experiencia de aprendizaje y la calidad de los MOOC. Campus Virtuales. Revista científica iberoamericana de tecnología educativa, 2 (2), 26-28.

Christensen, C., y Overdorf, M. (2000). Meeting the Challenge of Disruptive Change. Harvard Business Review, 78 (2), 67-77. 
Daniel, J. (2012). Making sense of MOOC: Musing in a mazr of myth, paradox and possibility. Journal of Interactive Media in Education, 18. doi: $10.5334 / 2012-18$.

Dennis, M. (2012). The Impact of MOOC on Higher Education. College and University Journal, 88 (2), 24-30.

DeWaard, I., Abajian, S., Gallagher, M., Hogue, R., Keskin, N., Koutropoulos, A., y Rodríguez, O. (2011). Using mLearning and MOOC to understand chaos, emergence, and complexity in education. International Review of Research in Open and Distance Learning, 12 (7), 94-115.

Duart, J. M., y Mengual-Andrés, S. (2014). Impact of the Knowledge Society in the University and in Scientific Communication. RELIEVE, $2 O$ (2), art. M4. doi: 10.7203/relieve.20.2.4343.

Gasevic, D., Kovanovic, V., Joksimovic, S., y Siemens, G. (2014). Where is research on massive open online courses headed? A data analysis of the MOOC Research Initiative. The International Review of Research in Open and Distance Learning, 15 (5).

Giorgetti, C. G., Romero, L., y Vera, M. (2013). Design of a specific quality assessment model for distance education. RUSC. Universities and Knowledge Society Journal, 10 (2), 301-315. doi: 10.7238/rusc. v10i2.1742.
Glance, D. G., Forsey, M., y Riley, M. (2013). The pedagogical foundations of massive open online courses. First Monday, 18 (5). doi: 10.5210/ fm.v18i5.4350.

Guàrdia, L., Maina, M., y Sangrà, A. (2013). MOOC Design Principles. A Pedagogical Approach from the Learner's Perspective. eLearning Papers, 33.

Kop, R., Fournier, H., y Mak, J. (2011). A pedagogy of abundance or a pedagogy to support human beings? Participant support on massive open online courses. International Review of Research in Open and Distance Learning, 12 (7), 74-93.

Lombillo Rivero, I., López Padrón, A., y Zumeta Izaguirre, E. (2012). Didactics of the use of ICT and traditionalteachingaidsinmunicipal higher education institutions. Journal of New Approaches in Educational Research, 1 (1), 33-40. doi: 10.7821/naer.1.1.33-40.

Mackness, J., Mak, S., y Williams, R. (2010). The ideals and reality of participating in a MOOC. En L. Dirckinck-Holmfeld, V. Hodgson, C. Jones, M. de Laat, D. McConnell y T. Ryberg (Eds.), Proceedings of the 7 th International Conference on Networked Learning 2010 (pp. 266-275). Lancaster: University of Lancaster.

Martín, O., González, F., y García, M. A. (2013). Propuesta de evaluación de la calidad de los MOOC a partir de 
la Guía Afortic. Campus Virtuales, 2 (1), 124-132.

McAuley, A., Stewart, B., Siemens, G., y Cormier, D. (2010). The MOOC model for digital practice. SSHRC Knowledge Synthesis Grant on the Digital Economy.

McMillan, J. H., y Shumacher, S. (2005) Investigación educativa. Madrid: Pearson- Adisson Wesley.

Najmul, A. (2013). Investigating e-learning system usage outcomes in the university context. Computers \& Education, 69, 387-399. doi: 10.1016/j.compedu.2013.07.037.

Pappano, L. (2012). The Year of the MOOC. The New York Times, 2 (12).

Prendes Espinosa, M. P., y Sánchez Vera, M. M. (2014). Arquímedes y la tecnología educativa: un análisis crítico en torno a los MOOC. Revista Interuniversitaria de Formación del Profesorado, 79, 29-49.

Read, T., y Rodrigo, C. (2014). Toward a Quality Model for UNED MOOC. eLearning Papers, 37, 43-50.

Rodrigo, C., Read, T., Santamaría, M., y Sánchez-Elvira, A. (2014). OpenupEdLabel for MOOC quality assurance: UNED COMA initial self-evaluation. En L. Bengochea Martínez, R. Hernández Rizzardini, y J. R. Hilera, (Eds.), Actas del $V$ Congreso Internacional sobre Calidad y Accesibilidad en la Formación Virtual (CAFVIR 2014). (pp. 551-555). Guatemala: Universidad Galileo.
Roig Vila, R., Mengual-Andrés, S., y Suárez Guerrero, C. (2014). Evaluación de la calidad pedagógica de los MOOC. Profesorado. Revista de currículum y formación del profesorado, 18 (1), 27-41.

Rosewell, J., y Jansen, D. (2014). The OpenupEd quality label: Benchmarks for MOOC. INNOQUAL, 2 (3), 88-100.

Sánchez Vera, M., Tomás Fernández, J., Serrano Sánchez, J., y Prendes Espinosa, M. (2013). Practical experiences for the development of educational systems in the semantic web. Journal of New Approaches in Educational Research, 2 (1), 23-31. doi:10.7821/naer.2.1.23-31-.

Skiba, D. J. (2012). Disruption in Higher Education: Massively Open Online Courses (MOOCs). Nursing Education Perspectives, 33 (6), 416417.

Torres Toro, S., y Ortega Carrillo, J. A. (2003). Indicadores de calidad en las plataformas de formación virtual: una aproximación sistemática. Etic@net, 1, 1-19.

Torres Mancera, D., y Gago Saldaña, D. (2014). Los MOOC y su papel en la creación de comunidades de aprendizaje y participación. RIED. Revista Iberoamericana de Educación a Distancia, 17 (1), 1334. doi: $10.5944 /$ ried.17.1.11570.

Vázquez, E., López, E., y Sarasola, J. (2013). La expansión del conocimiento en abierto: los MOOC. Barcelona: Editorial Octaedro. 
Vega Gil, L. (2013). Global politics and education systems: towards education markets? Journal of New Approaches in Educational Research, 2 (2), 95-101. doi:10.7821/ naer.2.2.95-101.

Vidal, A. A., y Camarena, B. O. (2014). Retos y posibilidades de los cursos en línea a partir de una experiencia concreta. Píxel-Bit, 44, 19-34. doi: 10.12795/pixelbit.2014.i44.02.

Williams, K., Kear, K., y Rosewell, J. (2012). Quality assessment for e-learning: $A$ benchmarking approach (2nd Ed.). Heerlen, The Netherlands: European Association of Distance Teaching Universities (EADTU).

Yousef, A. M. F., Chatti, M. A., Schroeder, U., Wosnitza, M., y Jakobs, H. (2014). MOOC: A Review of the State-of-the-Art. In CSEDU2014-6th International Conference on Computer Supported Education, 1-3 de abril. Barcelona, España. (pp. 9-20).

Yuan, L., y Powell, S. (2013). MOOC and Open Education: Implications for Higher Education. Cetis White Paper.

Zapata, M. (2013). MOOC, una visión crítica y una alternativa complementaria: La individualización del aprendizaje y de la ayuda pedagógica. Campus Virtuales. Revista científica iberoamericana de tecnología educativa. 1 (2), 20-38.

\section{PERFIL ACADÉMICO Y PROFESIONAL DE LOS AUTORES}

Santiago Mengual-Andrés. Doctor y Licenciado en Psicopedagogía. Profesor Ayudante Doctor del Dpto. de Educación Comparada e Historia de la Educación de la Facultad de Filosofía y Ciencias de la Educación de la Universidad de Valencia. Secretario Académico de la delegación de la Comunidad Valenciana de la Asociación Interuniversitaria de Investigación Pedagógica (AIDIPE). Editor ejecutivo del Journal of New Approches in Educational Research. Su investigación se centra en el análisis de Políticas nacionales e internacionales de inclusión TIC, Competencia Digital y nuevos escenarios de educación con tecnología. Ha participado y participa como docente en diversos másters y programas de posgrado de ámbito nacional e internacional. ORCID: http://orcid.org/0000-0002-1588-9741

E-mail: santiago.mengual@ua.es 
Rosabel Roig Vila. Rosabel Roig Vila, Doctora en Pedagogía, es Profesora Titular de Universidad del Dpto. de Didáctica General y Didácticas Específicas, directora del grupo de investigación EDUTIC-ADEI y directora del Máster en Educación y TIC de la Universidad de Alicante. Su investigación se centra en las TIC y los procesos de enseñanza-aprendizaje. Ha sido Decana de la Facultad de Educación de la Universidad de Alicante. Es editora de la Journal of New Approaches in Educational Research (http://www.naerjournal.ua.es). Ha sido investigadora en proyectos subvencionados con fondos de instituciones públicas españolas y de la Unión Europea. Es autora de un centenar publicaciones en libros, revistas y congresos de reconocido prestigio. Es miembro del equipo editorial científico de varias revistas científicas educativas. Es miembro del Consejo Asesor de varios centros de investigación superior y de comités científicos internacionales. Dirige desde el año 2003 el Máster "Educación y Tecnologías de la Información y la Comunicación”, título propio de la Universidad de Alicante. Participa en másters interuniversitarios (UOC, Universidad de Huelva, Universidad de Cádiz y Universitat de les Illes Balears) y diversos programas de doctorado. http://orcid.org/oooo-0002-9731-430X

E-mail: rosabel.roig@ua.es

\section{DIRECCIÓN DE LA AUTORA}

Department of Comparative Education \& History of Education, University of Valencia, Valencia, Spain

Avda. Blasco Ibáñez 30,

Faculty of Philosophy and Educational Sciences, Valencia (46010), Spain

Carmen Lloret Catalá. Profesora del Departamento de Educación Comparada e Historia de la Educación de la Universitat de València y miembro de la Unidad de Investigación en Política de la Educación (UINPE) de la Universitat de València. Ha trabajado durante ocho años como Técnico superior de investigación del Departamento de Psicología Básica de la Facultat de Psicologia de la Universitat de València. Ha participado como investigadora en numerosos proyectos de $\mathrm{I}+\mathrm{D}+\mathrm{i}$ y contratos y convenios de investigación con empresas privadas siendo la gestión de proyectos de $\mathrm{I}+\mathrm{D}+\mathrm{i}$ una de sus funciones. Sus líneas de investigación están relacionadas con la prevención, cambio de actitudes, diseño de programas de intervención, sensibilización y reeducación vial y el desarrollo y aplicación de las TIC en el ámbito educativo. En la actualidad está iniciando nuevas líneas de investigación sobre las competencias digitales en la educación, Mobile Learning y políticas educativas.

E-mail: carmen.lloret@uv.es 


\section{DIRECCIÓN DE LOS AUTORES}

Facultad de Educación.

Universidad de Alicante.

Campus Sant Vicent del Raspeig.

Ap. correos 99, 03080-Alicante, España

Fecha de recepción del artículo: 07/12/2014

Fecha de aceptación del artículo: 29/01/2015

\section{Como citar este artículo:}

Mengual-Andrés, S., Roig Vila, R., y Lloret Catalá, C. (2015). Validación del Cuestionario de evaluación de la calidad de cursos virtuales adaptado a MOOC. RIED. Revista Iberoamericana de Educación a Distancia, 18 (2), 145-169. doi: http://dx.doi.org/10.5944/ried.18.2.13664. 